\title{
OSTEOPOROSIS ASOCIADA A GLUCOCORTICOIDES, UNA APROXIMACIÓN PRÁCTICA. REVISIÓN
}

\author{
James Milthon Yurgaky S. MD. ${ }^{* *}$ y Ana María ArRedondo G. MD. ${ }^{2}$ \\ ${ }^{1}$ Internista, Residente Programa de Endocrinología, Universidad Militar Nueva Granada, \\ Bogotá, Colombia. ${ }^{2}$ Residente Programa de Medicina Interna, Universidad Militar Nueva \\ Granada, BOgotá, Colombia
}

\begin{abstract}
Resumen
La osteoporosis es una enfermedad de alta prevalencia en el mundo y una de las causas más importantes de su forma secundaria es el uso crónico de glucocorticoides; aproximadamente el 0,5\% de la población mundial es consumidora crónica de estos medicamentos, dadas sus múltiples indicaciones en el tratamiento de enfermedades reumáticas, dermatológicas, pulmonares o renales entre otras. Los pacientes con osteoporosis inducida por glucocorticoides presentan complicaciones como la fractura de cadera, situación que genera altos costos a los sistemas de salud. Por lo tanto, es muy importante hacer un diagnóstico rápido para iniciar un tratamiento oportuno, debido a que la prevención y el tratamiento de la enfermedad son menos costosos que el manejo de sus complicaciones. Se consideran en riesgo de osteoporosis todos aquellos pacientes que toman $5 \mathrm{mg}$ diarios de prednisolona o más, por un tiempo mínimo de tres meses; a estos pacientes se les debe realizar una densitometría ósea (DXA), que es el método diagnóstico de elección para todos los tipos de osteoporosis. Un $T$ Score diagnóstico para osteoporosis u osteopenia indican el inicio de tratamiento específico principalmente con bifosfonatos, entre los cuales el risedronato y alendronato han demostrado mejor efectividad. Otra de las indicaciones para el tratamiento específico estaría basada en el riesgo de fractura definido por la OMS con el Score FRAX. Finalmente, la prevención se debe hacer en todo paciente usuario crónico de glucocorticoides con medidas generales que implican cambios en el estilo de vida y la ingesta diaria de 1000 a $1500 \mathrm{mg}$ de calcio elemental y $600 \mathrm{u}$ de vitamina D.
\end{abstract}

Palabras clave: osteoporosis, glucocorticoides, toxicidad de medicamentos

\section{OSTEOPOROSIS ASSOCIATED WITH GLUCOCORTICOIDS, A PRACTICAL APPROACH. A REVIEW}

\begin{abstract}
Osteoporosis is a highly prevalent disease in the world and one of the most important causes of its secondary form is the chronic use of glucocorticoids; approximately $0.5 \%$ of the world's population is chronic consumer of this medicine because of its many indications in the treatment of rheumatic, dermatologic, pulmonary or renal diseases among others. Patients with glucocorticoid induced osteoporosis generates high costs for health systems derived from its complications, such as hip fractures, being more cost-effective the prevention and treatment of the disease than the management of its complications, that constitutes an important reason for its early diagnose and treatment. Every patient who takes prednisolone $5 \mathrm{mg}$ daily or more for a minimum of three months is considered at risk of glucocorticoid induced
\end{abstract}

* Correspondencia: James Yurgaky jamesy7656@yahoo.es. Dirección correspondencia: Tr. 3 No 49-00, Servicio de Endocrinología, Hospital Militar,Bogotá, Colombia. Teléfono: 4344842

Recibido: Octubre 22 de 2010 Aceptado: Diciembre 15 de 2010 
osteoporosis; these patients should be screened with DXA, which is the election diagnostic method for all types of osteoporosis. Diagnosis of osteoporosis or osteopenia by T score is indicative for specific treatment with biphosphonates, among which risedronate and alendronate have demonstrated to be more effective. Other indication for the treatment of these patients is based on the WHO's score FRAX. Finally, prevention must be done in every glucocorticoid chronic consumer, basically with changes in lifestyle and the daily intake of 1000-1500 mg of elemental calcium and 600 units of vitamin D.

Key words: osteoporosis, glucocorticoids, drug toxicity

\title{
OSTEOPOROSE ASSOCIADA COM GLICOCORTICÓIDES, UMA ABORDAGEM PRÁTICA. REVISÃO
}

\begin{abstract}
Resumo
A osteoporose é uma doença de alta prevalência no mundo e uma das causas mais importantes da forma secundária é o uso crônico de glicocorticóides; aproximadamente $0,5 \%$ da população mundial é usuária crônica destas drogas dadas as suas múltiplas indicações no tratamento de doenças reumáticas, pele, rim, pulmão e outros. Os doentes com osteoporose induzida por glucocorticóide apresentam complicações tais como fraturas de quadril que geram altos custos para os sistemas de saúde. Por isso é muito importante o diagnóstico precoce para dar tratamento imediato, devido a que são mais custo-efetiva que as suas complicações. São considerados de risco para osteoporose todos aqueles doentes que tomam $5 \mathrm{mg}$ de prednisolona por dia ou mais, por um período mínimo de três meses, esses pacientes devem ser submetidos a densitometria óssea (DEXA), que é considerado o método diagnóstico de escolha para todos os tipos de osteoporose. Um escore T para diagnosticar a osteoporose ou osteopenia indicam o início de um tratamento específico, principalmente, com bifosfonatos, incluindo o risedronato e alendronato mostraram maior eficácia. Outra indicação para o tratamento específico seria baseado no risco de fratura definida pelo Índice OMS com FRAX. Finalmente, a prevenção deve ser feita em todos os pacientes usuários crônicos de glucocorticoides com medidas gerais que envolvem mudanças no estilo de vida e ingestão diária de 1000-1500 mg de cálcio elementar e 600 U de vitamina D.
\end{abstract}

Palavras-chave: osteoporose, glucocorticoides, toxicidade de drogas

\section{Introducción}

La osteoporosis es una enfermedad frecuente que afecta en Norteamérica, alrededor de ocho millones de mujeres y dos millones de hombres $(1,2)$. Se produce como resultado final de diferentes procesos fisiopatológicos que generan una disminución de la mineralización de la matriz ósea, incrementando el riesgo de fracturas axiales y periféricas, contribuyendo de esta manera a un aumento de la mortalidad general, así como a un incremento en los costos derivados de las intervenciones quirúrgicas y de los cuidados institucionalizados de los pacientes con fractura de cadera, para el tratamiento integral de su enfermedad $(3,4,5)$.
Los glucocorticoides son un grupo de medicamentos de alta potencia antiinflamatoria y bajo costo, que son ampliamente utilizados para el tratamiento de múltiples enfermedades dermatológicas, intestinales, pulmonares, renales, reumatológicas y para evitar el rechazo en pacientes trasplantados; esto hace que una gran cantidad de personas alrededor del mundo, sean usuarios crónicos de glucocorticoides y sufran de los efectos adversos tales como la osteoporosis secundaria $(6,7)$.

Los efectos deletéreos de los corticoides sobre el hueso fueron descritos en conjunto con la enfermedad de Cushing alrededor de 1932; a pesar de ser los glu- 
cocorticoides ampliamente prescritos (en $0,5 \%$ de la población general), la búsqueda activa de la osteoporosis inducida por estos medicamentos no se realiza en todos los pacientes que son consumidores crónicos (8). En un estudio realizado en Nothinghan, Reino Unido, se encontró que de las 303 personas participantes que tomaban esta medicación, solo el $14 \%$ tenía implementada una estrategia para el diagnóstico temprano y reconocimiento de los trastornos de la densidad mineral ósea inducida por esteroides y menos del $6 \%$ recibió terapia preventiva específica $(9,10)$.

La dosis relacionada con efecto óseo deletéreo es usualmente $5 \mathrm{mg}$ de prednisolona diaria o su equivalente por más de tres meses (9-12). Se ha demostrado que el uso intermitente de ciclos cortos de glucocorticoides a dosis de hasta $15 \mathrm{mg}$ día de prednisolona en enfermedades crónicas como la artritis reumatoidea, no se ha relacionado con pérdida de masa mineral ósea (14). Durante los últimos años el interés por esta enfermedad ha venido creciendo; en la literatura hay varios estudios que indican claramente que para evitar en los pacientes el desarrollo de enfermedad mineral ósea derivada del empleo crónico de los glucocorticoides, se deben implementar medidas de prevención, diagnóstico y terapéutica (13). Hasta este momento los resultados de los estudios que ha evaluado el uso de esteroides por vía inhalada, no han dado una idea clara sobre el impacto que tiene sobre el hueso $(15,16)$.

\section{Fisiopatología}

La interacción entre esteroides y hueso es compleja; múltiples mecanismos se han relacionado con la pobre calidad mineral ósea que dan como resultado la disminución en la formación ósea e incremento de la resorción, entre otros su efecto directo sobre las células óseas, disminuyendo la producción de osteoblastos y osteoclastos, aumentando la apoptosis de osteoblastos y disminuyendo el tiempo de vida de los osteoclastos (figura 1) (17). Los glucocorticoides ejercen su efecto en el hueso mediante mecanismos directos e indirectos; entre los indirectos, los cuales pueden generar un aumento en la pérdida de la masa ósea y por lo consiguiente, un riesgo mayor en las caídas están: la disminución de la absorción de calcio en el intestino, el aumento de la excreción renal de calcio, la disminución de la acción de los esteroides sexuales y de la hormona de crecimiento, la disminución de la fuerza muscular y las alteraciones en la interacción musculoesquelética (18).

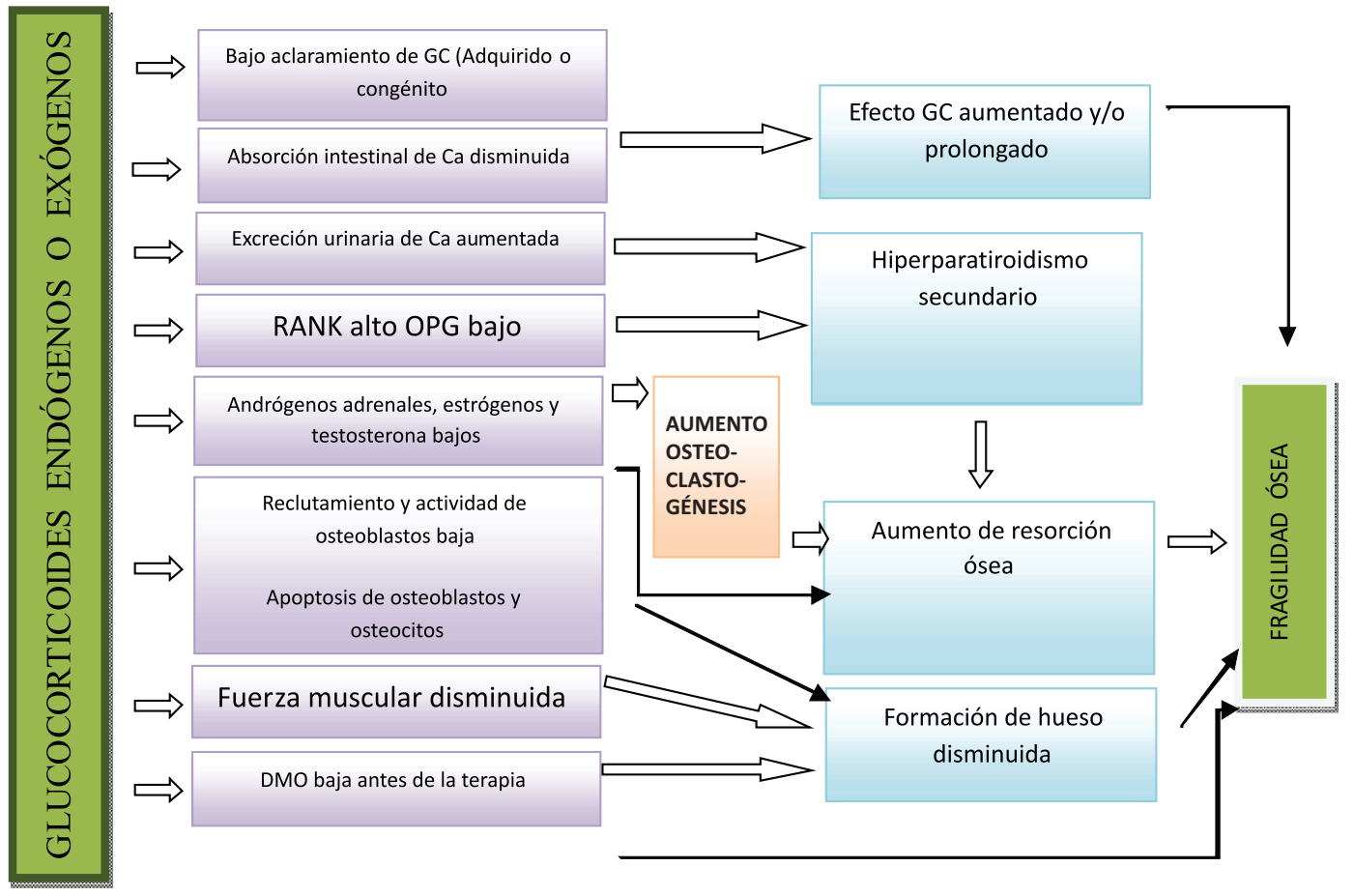

Figura 1. Fisiopatología del hueso. GC: Glucocorticoides, RANK: receptor activador del ligandoNF-KB, OPG: osteoproteregina, DMO: Densidad mineral ósea, (17) 
En los estudios realizados por Abu y col. y por Beavan $y$ col., en los que se utilizaron anticuerpos policlonales de alta afinidad, se identificó la presencia de receptores específicos citoplasmáticos en los osteoblastos denominados receptores funcionales alfa de glucocorticoides, los cuales al unirse a su ligando, activan la vías de señalización que generan una disminución en la producción local de hueso nuevo $(19,20)$

Gracias a los estudios histomorfométricos se ha establecido con claridad, en pacientes expuestos crónicamente al uso de glucocorticoides, una disminución en la formación de nuevo hueso $(21,22)$; de igual manera, al evaluar la viabilidad de los osteocitos se observó una disminución de la cantidad de células viables y un aumento de la apoptosis en estos pacientes, cuando se comparaban con individuos sanos $(23,24)$. Los osteoclastos maduros no expresan receptores citoplasmáticos de corticoides; sin embargo, a través de la supresión de osteoprotegerina y del incremento del factor de transcripción nuclear $\mathrm{NF}_{\mathrm{K}} \mathrm{B}$, altas dosis de glucocorticoides son capaces de inducir la diferenciación de nuevos osteoclastos a partir de la línea monocito macrófago $(25,26)$.

Por otro lado, los esteroides son capaces de disminuir las concentraciones corporales de calcio, al disminuir hasta en un $30 \%$ del valor basal la absorción intestinal al día catorce de administración de prednisolona, e incrementar el valor de excreción renal de calcio hasta dos veces después de cinco días de medicación, esto independiente de la vitamina $\mathrm{D}$ y sus metabolitos activos $(23,24)$. Al final, todos estos procesos generan una fragilidad notable del sistema esquelético que da como resultado un riesgo estimado mayor de fractura vertebral $(\mathrm{RR}=2,86$; IC $95 \%, 2,56-3,16)$ y de fractura de cadera $(\mathrm{RR}=2,01$; IC 95\%, 1,74-2,29) comparado con la población que no usa este tipo de medicación $(27,28)$. No solo el uso crónico de esteroides es el que facilitan la pérdida de la masa mineral ósea; también la inducen algunos polimorfismos genéticos, como en el caso de los pacientes con insuficiencia adrenal (Enfermedad de Addison), en quienes se demostró que el polimorfismo C3435T de la glicoproteína $\mathrm{P}$ transportador se asocia con aumento en la sensibilidad a los glucocorticoides y a la pérdida mineral ósea secundaria, sobre todo cuando se usan dosis de hidrocortisona mayores a $25 \mathrm{mg}$ día. (29)

\section{Riesgo de fractura}

La presentación de fracturas vertebrales y no vertebrales en pacientes sometidos a uso crónico de esteroides está en relación con varios factores; el primero de ellos es el tiempo de uso del medicamento, así como la dosis recibida del mismo; ingerir glucocorticoides por un año consecutivo, incrementa el riesgo de fractura vertebral hasta 5,9 veces y de cadera hasta 5,0 veces, con aumento acumulado del porcentaje durante el primer año de fractura vertebral sintomática o no en un $54 \%$ (31). De acuerdo a la dosis recibida se puede discriminar el riesgo de cualquier fractura de la siguiente manera $(32,33)$ :

- Prednisolona 2,5-7,5 mg v.o. día: razón de riesgo 1,77

- Prednisolona > 7,5 mg v.o. día: razón de riesgo 2,27

Por otro lado, es importante recalcar que la presencia de un determinado valor de densidad mineral ósea, no se relaciona directamente con un menor riesgo de fractura; el Dr. Hiroshi KAJI pudo demostrar en mujeres quienes habían usado en forma crónica glucocorticoides, que el umbral para la presentación de fracturas relacionado con la densidad mineral ósea medida por densitometría ósea, es más alto en aquellos pacientes que sufren fracturas comparado con los que no, $0,807 \mathrm{~g} / \mathrm{cm}^{3}$ vs $0,716 \mathrm{~g} / \mathrm{cm}^{3}$ en columna; $0,61 \mathrm{~g} / \mathrm{cm}^{3}$ vs $0,581 \mathrm{~g} / \mathrm{cm}^{3}$ en femur y 0,592 $\mathrm{g} / \mathrm{cm}^{3}$ vs $0,477 \mathrm{~g} / \mathrm{cm}^{3}$ en radio respectivamente $(32,33)$.

\section{Diagnóstico específico}

En el examen físico se pueden observar ciertas características que pueden indicar con alta especificidad que el paciente ha estado expuesto en forma crónica a los glucocorticoides; dentro de estos se destacan la cara de luna llena, sangrado fácil, adelgazamiento cutáneo y la miopatía proximal $(30,31)$. En este contexto, la sospecha de efectos deletéreos en la densidad mineral ósea es mucho más alta si se compara con la mayoría de los pacientes que usan glucocorticoides y que no tienen este tipo de características; por esta razón, se han diseñado protocolos para la detección temprana de osteopenia y osteoporosis.

La histomorfometría sigue siendo la prueba de oro para el diagnóstico de la osteoporosis $(32,33)$, pero como es un procedimiento invasivo con una alta morbilidad, la organización mundial de la salud (OMS) ha abalado a la densitometría ósea (DXA) como método para la búsqueda de osteoporosis independiente de la etiología que la origine $(34,35,36)$; se ha definido que el punto de corte para el $T$ score menor de -1,0 y -2,5, establece el diagnóstico de osteopenia y osteoporosis respectivamente. En general, a los pacientes que tengan historia de uso crónico de esteroides o que vayan a recibirlos por más 
de tres meses se les debe realizar una densitometría y si el resultado es normal o se diagnostica una osteoporosis o una osteopenia se debe hacer un seguimiento cada tres años o anual o bianual respectivamente $(35,36)$.

Aunque estas medidas deben estar bien establecidas en los programas de usuarios crónicos de corticoides, es interesante anotar que los estudios realizados en pacientes en riesgo y con alteraciones de la densidad mineral ósea han mostrado que la indicación de la densitometría solo se hace en el 5 al $12 \%$ de los pacientes (37). Los resultados de los estudios sobre programas de educación para pacientes y médicos tratantes de usuarios crónicos de corticoides han sido divergentes, ya que algunos han mostrado un aumento en la solicitud de exámenes para el diagnóstico temprano y la prescripción de medicamentos para la prevención temprana de la osteoporosis, mientras que otros, no han mostrado ningún cambio en el comportamiento de los pacientes ni en el de los médicos tratantes $(38,39)$.

Estudios transversales y longitudinales en los que se compararon pacientes en terapia con glucocorticoides vs controles sanos, se documentó un incremento del riesgo de fractura en el primer grupo, a pesar de tener densidad mineral ósea más alta, $(32,33,40,41)$. Otros métodos tales como la ecografía de calcáneo, tomografía computarizada cuantitativa y los marcadores de resorción ósea, tienen una utilidad limitada debido a una ausencia notable de criterios uniformes que no permiten estandarizar el diagnóstico $(42,43)$.

\section{Prevención}

Los dos objetivos primordiales deben orientarse hacia la prevención de fracturas vertebrales y no vertebrales, así como a la disminución de la tasa de pérdida de hueso que se genera una vez se instaura el tratamiento con glucocorticoides (44). Las medidas generales incluyen una dieta rica en calcio elemental que garantice un consumo diario entre 1000 a $1500 \mathrm{mg}$, con un suplemento de vitamina D de 600 UI para todos los pacientes consumidores de esteroides por más de tres meses $(45,46)$; adicionalmente se recomienda realizar ejercicio regular por lo menos 150 minutos semanales, lo cual ha demostrado que reduce la absorción de los glucocorticoides orales y por lo tanto se cree, puede disminuir sus efectos adversos (47).

También se recomienda la abstinencia de tabaco, disminución del consumo de alcohol y café y aumento de esfuerzos encaminados para disminuir las caídas en personas con factores de riesgo $(45,46)$. El uso de la dosis mínima de corticoide necesaria para el tratamiento disminuiría la presentación de la osteoporosis en algunos pacientes, ya que la pérdida de masa mineral ósea se asocia con dosis mayores a $5 \mathrm{mg}$ diarios de prednisona por más de tres meses, dosis que se podría reducir en algunos pacientes que así lo toleren (48).

\section{Indicaciones de tratamiento}

Inicialmente hubo controversia respecto del momento de inicio del tratamiento en este tipo de pacientes; actualmente se ha llegado a un consenso gracias a los resultados que se reportan en la literatura, de los diferentes métodos que se han usado para determinar el momento propicio para el inicio de intervenciones terapéuticas (31); por ejemplo, en mujeres postmenopáusicas y hombres mayores de 50 años se usa el FRAX (herramienta de evaluación de riesgo de fractura) que ha sido recientemente desarrollada por la OMS y es útil para decidir el inicio de tratamiento específico (Tabla 1); por otro lado, usando las escalas de puntuación obtenidas por análisis multivariado se puede determinar el riesgo de fractura a cinco años; así los valores de 30,40 y 50 puntos indican un riesgo de $6,2 \%, 15,2 \%$ y $35,2 \%$ respectivamente, para los pacientes que son usuarios de glucocorticoides (Tabla 2) (31).

En general se recomienda iniciar el tratamiento específico en las siguientes situaciones clínicas:

Tabla 1. Frax

\begin{tabular}{|l|c|c|}
\hline \multicolumn{3}{|c|}{ "Herramienta de evaluación de riesgo de fractura" } \\
\hline Edad & Mujer & Hombre \\
\hline Sexo & & \\
\hline Peso $(\mathrm{Kg})$ & & \\
\hline Estatura $(\mathrm{cm})$ & $\mathrm{No}$ & $\mathrm{Si}$ \\
\hline Fractura previa & $\mathrm{No}$ & $\mathrm{Si}$ \\
\hline Fractura de cadera de un padre & $\mathrm{No}$ & $\mathrm{Si}$ \\
\hline Fumador habitual & $\mathrm{No}$ & $\mathrm{Si}$ \\
\hline Glucocorticoides & $\mathrm{No}$ & $\mathrm{Si}$ \\
\hline Artritis reumatoidea & $\mathrm{No}$ & $\mathrm{Si}$ \\
\hline Osteoporosis secundaria & $\mathrm{No}$ & $\mathrm{Si}$ \\
\hline 3 o mas unidades de alcohol al día & No & $\mathrm{Si}$ \\
\hline Densidad mineral ósea femoral $\left(\mathrm{g} / \mathrm{cm}^{2}\right)$ & & \\
\hline
\end{tabular}

Riesgo de fractura a diez años mediante correlación de Poisson $(49,59)$

Tratamiento si:

- Riesgo de fractura de cadera mayor al $3 \%$

- Riesgo de fractura general mayor a $20 \%$ 
Tabla 2. Score para predecir riesgo de fractura

\begin{tabular}{|c|c|c|c|c|c|c|c|c|c|}
\hline \multirow[b]{2}{*}{ EDAD } & \multicolumn{3}{|c|}{$\begin{array}{c}\text { TODAS LAS } \\
\text { FRACTURAS }\end{array}$} & \multicolumn{3}{|c|}{ CADERA } & \multicolumn{3}{|c|}{ COLUMNA } \\
\hline & 50 & 65 & 80 & 50 & 65 & 80 & 50 & 65 & 80 \\
\hline 7,5 mg día & 8 & 6 & 5 & 12 & 8 & 4 & 15 & 14 & 12 \\
\hline 15mg día & 11 & 9 & 7 & 15 & 10 & 5 & 20 & 18 & 16 \\
\hline Edad por cada 10 años & 4 & 4 & 4 & 8 & 8 & 8 & 4 & 4 & 4 \\
\hline Hombre & \multicolumn{3}{|c|}{-6} & \multicolumn{3}{|c|}{-6} & \multicolumn{3}{|c|}{-4} \\
\hline $\mathrm{IMC}<20$ & \multicolumn{3}{|c|}{3} & \multicolumn{3}{|c|}{6} & \multicolumn{3}{|c|}{3} \\
\hline $\mathrm{IMC}>26$ & \multicolumn{3}{|c|}{-1} & \multicolumn{3}{|c|}{-4} & \multicolumn{3}{|c|}{-1} \\
\hline Fumador & \multicolumn{3}{|c|}{1} & \multicolumn{3}{|c|}{2} & \multicolumn{3}{|c|}{1} \\
\hline Caĺda en seis meses previos & \multicolumn{3}{|c|}{8} & \multicolumn{3}{|c|}{7} & \multicolumn{3}{|c|}{6} \\
\hline Fractura previa uso esteroides & \multicolumn{3}{|c|}{0} & \multicolumn{3}{|c|}{4} & \multicolumn{3}{|c|}{5} \\
\hline $\begin{array}{l}\text { Fracturas accidentales con uso } \\
\text { de GC }\end{array}$ & \multicolumn{3}{|c|}{0} & \multicolumn{3}{|c|}{4} & \multicolumn{3}{|c|}{5} \\
\hline $\begin{array}{l}\text { Hospitalización reciente por indi- } \\
\text { cación de GC }\end{array}$ & \multicolumn{3}{|c|}{4} & \multicolumn{3}{|c|}{4} & \multicolumn{3}{|c|}{9} \\
\hline \multicolumn{10}{|l|}{ INDICACIÓN } \\
\hline $\mathrm{AR}$ & \multicolumn{3}{|c|}{1} & \multicolumn{3}{|c|}{4} & \multicolumn{3}{|c|}{3} \\
\hline Colitis no infecciosa & \multicolumn{3}{|c|}{1} & \multicolumn{3}{|c|}{2} & \multicolumn{3}{|c|}{3} \\
\hline
\end{tabular}

GC: Glucocorticoides

Riesgo relativo de fractura a cinco años de acuerdo al score: 30: 6,2\%; 40: 15,3\%; 50: 35,2\% (31)

1. Pacientes con diagnóstico de osteopenia con un $T$ score menor de $-1,0 \mathbf{y}$ que hayan recibido por más de tres meses prednisolona a dosis de $5 \mathrm{mg}$ día o su equivalente (31).

2. Todos aquellos pacientes en quienes se documente osteoporosis (T score menor a -2,5) $(49,50)$.

3. Cálculo de riesgo de fractura por score de FRAX superior a $3 \%$ para cadera y más de $20 \%$ para cualquier tipo de fractura.

\section{Costoefectividad del tratamiento}

El costo de esta enfermedad para los sistemas de salud locales, así como el impacto que tienen las medidas terapéuticas en la prevención de fracturas y la calidad de vida de los pacientes, analizados en el contexto de la farmacoeconomía, son una herramienta útil para el médico al justificar aun más los esfuerzos encaminados a la detección y tratamiento tempranos de las alteraciones metabólicas óseas en pacientes con uso crónico de glucocorticoides $(4,5,51,52)$.

En mujeres tratadas con esteroides, el uso de vitamina D y calcio reduce la presentación de fracturas en un
$40 \%$ a 10 años, con un costo aproximado de 800 dólares por fractura evitada; los costos generados por el etidronato y el alendronato son más altos, son alrededor de los 2000 y 3000 dólares respectivamente $(51,52)$. Por cada año de calidad de vida ganado en pacientes tratados con $5 \mathrm{mg}$ de prednisolona diaria, se invierten alrededor de 68 dólares anuales; a dosis más altas de $15 \mathrm{mg}$ diarios, el costo de cada año de vida ganado se incrementa.

El uso de un bifosfonato cuesta en promedio 471 dólares anuales; sin embargo, esto resulta poco si se compara con el costo del tratamiento para pacientes que sufren fracturas osteoporóticas de cadera, el cual puede ser equivalente a unos 51000 dólares durante el primer año y 39000 dólares anuales en todas aquellas personas que requieren a una tercera persona para su cuidado después del evento inicial $(4,5)$. En general, los costos generados por el tratamiento de los diferentes tipos de fracturas, así como lo requerido para la prevención de las mismas, está determinado por cuatro factores importantes que son: la edad del paciente, la capacidad de auto cuidado del mismo, la dosis de esteroide recibida y la localización de la fractura $(4,5)$. 


\section{¿Con qué tratar?}

Existe un número representativo de estudios que han evaluado los diferentes grupos farmacológicos, para la prevención de riesgo de fractura vertebral y no vertebral en pacientes sometidos a uso crónico de esteroides; en general, los medicamentos que cuentan con mejor evidencia clínica son los antirresortivos, siendo el risedronato en primer lugar y el alendronato como segunda opción los que más fortaleza y mejores desenlaces han mostrado; por otro lado, medicamentos como el raloxifeno y el ranelato de estroncio, no cuentan con estudios aleatorizados prospectivos que permitan valorar su utilidad en la prevención y tratamiento de la osteoporosis inducida por esteroides.

Recientemente se aprobó el ácido zoledrónico para el tratamiento de osteoporosis inducida por esteroides basados en la información obtenida de un estudio multicéntrico, randomizado, doble ciego de no inferioridad que comparó esta molécula con el risedronato y se observó que el incremento de la DMO fue de 2,06 $\%$ vs $0,64 \%$ obtenido con el risedronato; también se evidenció una menor presentación de fracturas vertebrales en el grupo de pacientes tratados con ácido zoledrónico vs risedronato (71).

Existen varios estudios en modelos animales con análogos de la hormona paratifoidea, que en teoría tiene ventajas para el tratamiento de la osteoporosis inducida por corticosteroides, ya que su mecanismo de acción en la fisiología ósea es contrarrestar el efecto que ejercen los corticoides; sin embargo en el campo clínico, existen solo estudios pequeños y con un seguimiento no mayor a 18 meses, en los que se demuestra que teriparatide tiene una ventaja sobre el alendronato en cuanto al aumento de la densidad mineral ósea, en donde hay una diferencia significativa en fracturas de cadera y con disminución en la tasa de fracturas vertebrales a dos años, pero no hay diferencias significativas en otro tipo de fracturas (69). Algo similar ocurre con la terapia de suplencia hormonal, el fluoruro sódico, la calcitonina y los metabolitos de la vitamina $\mathrm{D}$ con los que no se ha observado una disminución en la tasa de fracturas en los estudios de valoración (51-59). En la tabla 3 se registran algunos de los estudios que han evaluado diferentes fármacos utilizados para el tratamiento de osteoporosis en estos pacientes.
El denosumab es un anticuerpo monoclonal contra el RANKL (ligando del receptor activador del factor nuclear ${ }_{K} B$ ), aprobado por la FDA para el tratamiento de osteoporosis posmenopáusica; sin embargo, en osteoporosis inducida por esteroides no hay suficiente información que permita recomendar su uso.

Tabla 3. Estudios que evaluan fármacos para la osteoporosis

\begin{tabular}{c} 
Referencia $\begin{array}{c}\text { Datos sobresalientes del estudio sobre } \\
\text { los desenlaces medidos }\end{array}$ \\
\hline
\end{tabular}

Estudio de 224 pacientes divididos en tres grupos (todos con suplemento de calcio) y se evaCohen S. luó: risedronato $5 \mathrm{mg} /$ día. $2,5 \mathrm{mg} /$ día y placebo. 1999 (60) Resultado: una menor tasa de fractura vertebral en el grupo de risedronato $5 \mathrm{mg}$ al comparar con placebo $(5,7 \%$ vs $17,1 \%)$ así como de las no vertebrales $3,9 \%$ vs $5,2 \%$ respectivamente.

\begin{tabular}{|c|c|}
\hline $\begin{array}{l}\text { Reid. } \\
2000 \text { (61) }\end{array}$ & $\begin{array}{l}\text { Incluyó } 290 \text { pacientes en tres grupos: risedro- } \\
\text { nato } 5 \mathrm{mg} / \text { día, } 2,5 \mathrm{mg} / \text { día y placebo (todos } \\
\text { suplementados con calcio } 1 \text { gr y vitamina D } 800 \\
\text { UI); al comparar combinados los dos grupos de } \\
\text { risedronato se observo una reducción del } 70 \\
\% \text { de riesgo de fractura vertebral Vs placebo. }\end{array}$ \\
\hline $\begin{array}{l}\text { Yuichi } \\
\text { Kikuchi } \\
2007(62)\end{array}$ & $\begin{array}{l}\text { Valoró } 38 \text { pacientes con enfermedad glomerular } \\
\text { y uso crónico de esteroides divididos en tres gru- } \\
\text { pos: risedronato } 2,5 \mathrm{mg} / \text { día vo, alfacalcidiol } 0,5 \\
\text { mg/día y la combinación de estos dos agentes } \\
\text { a las dosis mencionadas; el grupo de risedro- } \\
\text { nato solo y combinado con alfacalcidiol mostró } \\
\text { incremento de la densidad mineral ósea hasta } \\
2,1 \% \text { vs alfacalcidiol solo que mostró descenso } \\
\text { de la DMO en - } 5,6 \%\end{array}$ \\
\hline
\end{tabular}

Comparación de alendronato 10mg/día vo + placebo vs alfacalcidiol $1 \mathrm{mg}$ vo día + placebo

Ron N.J. en un total de 200 pacientes. El grupo de bifosRon N.J. fonato incrementó la densidad mineral ósea en de $\mathrm{Nijs}$ (63) $2,1 \%$ vs $-1,9 \%$ para calcidiol y en cadera 2006 (63) $\quad 1,6 \%$ vs $-2,0 \%$; tres fracturas vertebrales nuevas se registraron en el grupo de alendronato vs. ocho en el grupo placebo.

Valoró la capacidad del alendronato en 208 pacientes para disminuir la pérdida de masa mineral ósea durante un tiempo de dos años a dosis de $10 \mathrm{mg} /$ día vo vs $2,5 \mathrm{mg} /$ día vo vs 2,5 $10 \mathrm{mg}$ intercalado día vs placebo. En todos los

Adachi JD. casos se suplementó con calcio y vitamina D; $\begin{array}{ll}\text { Adachi JD. } & \text { el grupo de } 10 \mathrm{mg} \text { de bifosfonato incrementó }\end{array}$ la masa mineral ósea espinal hasta un $3,9 \%$ vs pérdida de $-0,8 \%$ para el grupo placebo, presencia de fracturas vertebrales y no vertebrales $0,7 \%, 5.4 \%$ vs $6,8 \%, 9,8 \%$ respectivamente, sin diferencias respecto a la seguridad de los pacientes. 


\begin{tabular}{ll}
\hline & 102 Pacientes asignados a dos grupos: etidrona- \\
& to 200 mg/día por 14 días cada 90 días $+3 \mathrm{gr}$ \\
& de calcio $+0,75$ mg de calcidiol diarios vs 3 gr \\
& de calcio mas 0,75 mg de calcidiol diarios; se \\
Sato & presentaron dos fracturas vertebrales en el gru- \\
po sin bifosfonato vs ninguna con bifosfonato, el \\
cual además incrementó la masa mineral ósea \\
hasta en un 10,1\% en mujeres postmenopáusi- \\
cas vs +1,4 \% para el grupo placebo.
\end{tabular}

\section{Conclusiones}

1. La osteoporosis asociada a glucocorticoides es una enfermedad frecuente y subdiagnosticada.
2. Todo paciente con uso de prednisolona $5 \mathrm{mg}$ día vo o su equivalente por más de tres meses debe recibir en promedio calcio elemental $1200 \mathrm{mg}$ diarios y vitamina D $600 \mathrm{u}$ al día.

3. Los pacientes a quienes por su enfermedad de base se deba suministrar tratamiento por más de tres meses con glucocorticoides sistémicos a dosis de prednisolona $5 \mathrm{mg} /$ día vo, deben tener una densitometría inicial, una anual como control si se diagnostica osteoporosis, bianual si se diagnostica osteopenia y cada tres años si esta dentro de límites normales.

4. Es aconsejable realizar una densitometría basal en todo paciente que tenga historia de uso de glucocorticoide de manera crónica y hacer seguimiento de acuerdo al diagnóstico densitométrico.

5. Pacientes con uso crónico de esteroides y diagnóstico densitométrico de osteopenia, con T score inferior a -1, ameritan tratamiento con bifosfonato.

6. Pacientes con diagnóstico de osteoporosis dado por $\mathrm{T}$ score inferior a $-2,5$, deben recibir tratamiento con bifosfonato.

7. Dentro de los medicamentos estudiados para el tratamiento y la prevención de la osteoporosis asociada a glucocorticoides, los que cuentan con un estudio más extenso que valoró riesgo de fractura así como impacto sobre la densidad mineral ósea, son de primera elección el risedronato y en segunda instancia el alendronato.

8. Medicamentos como la calcitonina, ranelato de estroncio, fluoruro de calcio y la terapia de suplencia hormonal, no tienen estudios que respalden el uso de los mismos para la prevención y tratamiento de la enfermedad ósea inducida por glucorticoides.

El flujograma de la figura 2 resume las acciones que se deben tomar para el diagnóstico y el tratamiento de la osteoporosis inducida por glucocorticoides.

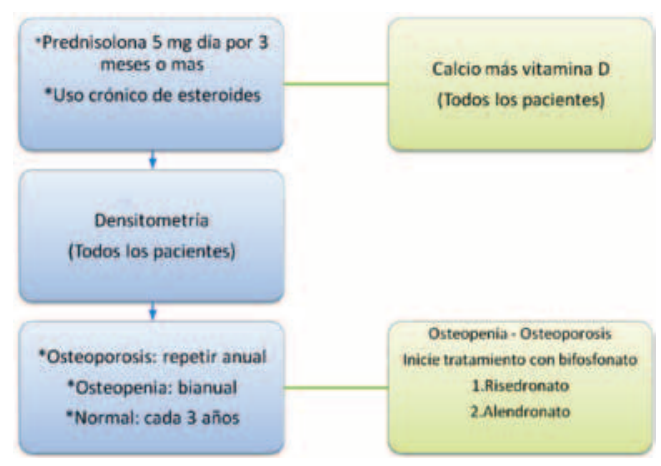

Figura 2. Flujograma de medidas de acción para diagnóstico y tratamiento de osteoporosis 


\section{Referencias}

1. Chapurlat R, Gamero P, Sornay-Rendu E, Arlot M, Claustrat B, Delmas P. Longitudinal study of bone loss in pre and perimenopausal women: evidence for bone loss in perimenopausal women. Osteoporos Int. 2000; 11(6):493-8.

2. Lindsay R, Nieves J, Golden A, Kelsey J. Bone mass among premenopausal women. Int J Fertil Menopausal Stud. 1993;38 Suppl 2:83-7.

3. National Osteoporosis Foundation. Physician's guide to prevention and treatment of osteoporosis. 2008 [Citado oct 2010]. Disponible en http://www.nof.org/professionals/Clinicians Guide.htm.

4. Van Staa TP, Geusens P, Zhang B, Leufkens HGM, Boonen A, Cooper $\mathrm{C}$. Individual fracture risk and the cost-effectiveness of bisphosphonates in patients using oral glucocorticoids. Rheumatology. 2007; 46(3):460-466.

5. Van Staa TP, Kanis JA, Geusens P, Boonen A, Leufkens HG, Cooper C. The cost-effectiveness of bisphosphonates in postmenopausal women based on individual long-term fracture risks. Value Health. 2007;10(5):348-57.

6. Summey BT, Yosipovitch G. Glucocorticoid-induced bone loss in dermatologic patients: an update. Arch Dermatol. 2006;142(1):82-90.

7. Dore RK. How to prevent glucocorticoid-induced osteoporosis. Cleve Clin J Med. 2010;77(8):529-36.

8. Guzman-Clark JR, Fang MA, Sehl ME, Traylor L, Hahn TJ. Barriers in the management of glucocorticoid-induced osteoporosis. Arthritis Rheum. 2007;57(1):140-6.

9. Walsh L, Wong C, Pringle M, Tattersfield A. Use of oral corticosteroids in the community and the prevention of secondary osteoporosis: a cross sectional study. BMJ. 1996; 313 (7053): 344-6.

10. Smith MD, Cheah SP, Taylor K, Ahern MJ. Prevention of corticosteroid induced osteoporosis in inpatients recently discharged from a tertiary teaching hospital. J Rheumatol. 2001;28(3):566-70.

11. VanStaa T, Leufkens M, Abenhaim L, Zhang B, Cooper C. Oral corticosteroid and fracture risk: relationship to daily and cumulative doses. Rheumatology. 2000; 39 (12):1383-1389

12. Donnan PT, Libby G, Boyter AC, Thompson P. The population risk of fractures attributable to oral corticosteroids. Pharmacoepidemiol Drug Saf. 2005 Mar;14(3):177-86.

13. American College of Rheumatology Task Force on osteoporosis Guidelines. Recommendations for the prevention and treatment of glucocorticoid induced osteoporosis. Arthritis Care Res. 1996: 39 (11):1791-801.

14. Ibañez M, Ortiz AM, Castrejón I, García-Vadillo JA, Carvajal I, Castañeda S, González-Alvaro I. A rational use of glucocorticoids in patients with early arthritis has a minimal impact on bone mass. Arthritis Res Ther. 2010;12(2):R50.

15. Israel E, Banerjee TR, Fitzmaurice GM, Kotlov TV, LaHive K, LeBoff MS. Effects of inhaled glucocorticoids on bone density in premenopausal women. N Engl J Med. 2001: 345(13): 941-7.

16. Yanik B, Ayrim A, Ozol D, Koktener A, Gokmen D. Influence of obesity on bone mineral density in postmenopausal asthma patients undergoing treatment with inhaled corticosteroids. Clinics (Sao Paulo). 2009;64(4):313-8.

17. Devogelaer JP. Glucocorticoid induced osteoporosis: Mechanisms and therapeutic approach. Rheum Dis Clin N Am. 2006;32:733-57

18. Hofbauer LC, Rauner M. Minireview: live and let die: molecular effects of glucocorticoids on bone cells. Mol Endocrinol. 2009;23(10):1525-31.
19. Abu EO, Horner A, Kusec V, Triffitt JT, Compston JE. The localization of the functional glucocorticoid receptor alpha in human bone. J Clin Endocrinol Metab. 2000: 85(2):883-9.

20. Beavan S, Horner A, Bord S, Ireland D, Compston J. Colocalization of glucocorticoid and mineralocorticoid receptors in human bone. J Bone Miner Res. 2001;16(8):1496-504.

21. Chappard D, Legrand E, Basle MF, Fromont P, Racineux JL, Rebel A, et al. Altered trabecular architecture induced by glucocorticoids: a bone histomorphometric study. J Bone Miner Res 1996:11(5):676-85.

22. Legrand E, Audran M, Guggenbuhl P, Levasseur R, Chalès G, Baslé MF, Chappard D. Trabecular bone microarchitecture is related to the number of risk factors and etiology in osteoporotic men. Microsc Res Tech. 2007;70(11):952-9.

23. Sambrook PN, Hughes DR, Nelson AE, Robinson BG, Mason RS. Osteocyte viability with glucocorticoid treatment: relation to histomorphometry. Ann Rheum Dis. 2003;62(12):1215-7.

24. LoCascio V, Ballanti P, Milani S, Bertoldo F, LoCascio C, Zanolin EM, Bonucci E. A. Histomorphometric long-term longitudinal study of trabecular bone loss in glucocorticoidtreated patients: prednisone versus deflazacort. Calcif Tissue Int. 1998;62(3):199-204.

25. Canalis E. Clinical review 83: Mechanisms of glucocorticoid action in bone: implications to glucocorticoid-induced osteoporosis. J Clin Endocrinol Metab. 1996;81(10):3441-7

26. Canalis E, Delany AM. Ann N Y Acad Sci. Mechanisms of glucocorticoid action in bone. 2002;966:73-81.

27. VanStaa TP, Leufkens HG, Cooper C. The epidemiology of corticosteroid induced osteoporosis: a meta-analysis. Osteoporos Int. 2002;13(3):777-87.

28. Walsh LJ, Lewis SA, Wong CA, Cooper S, Oborne J, Cawte SA, Harrison T, Green DJ, Pringle M, Hubbard R, Tattersfield $\mathrm{AE}$. The impact of oral corticosteroid use on bone mineral density and vertebral fracture. Am J Respir Crit Care Med. 2002;166(5):691-5.

29. Løvås K, Gjesdal CG, Christensen M, Wolff AB, Almås B, Svartberg J, Fougner KJ, Syversen U, Bollerslev J, Falch JA, Hunt PJ, Chatterjee VK, Husebye ES. Glucocorticoid replacement therapy and pharmacogenetics in Addison's disease: effects on bone. Eur J Endocrinol. 2009;160(6):993-1002.

30. Nieman LK, Biller BM, Findling JW, Newell-Price J, Savage MO, Stewart PM, Montori VM. The Diagnosis of Cushing's Syndrome: An Endocrine Society Clinical Practice Guideline. J Clin Endocrinol Metab. 2008; 93(5): 1526-40.

31. VanStaa TP, Geusens P, Pols HA, Laet H, Leufkens HG, Cooper C. A simple score for estimating the long-term risk of fracture in patients using oral glucocorticoids. QJM. 2005; 98(3):191-8.

32. Kaji H, Mamauchi M, Chihara K, Sugimoto T. The Threshold of Bone Mineral Density for Vertebral Fracture in Female Patients with Glucocorticoid-induced Osteoporosis. Endocr J. 2006; 53(1): 27-34.

33. Kaji H, Tobimatsu T, Naito J, Iu MF, Yamauchi M, Sugimoto T, Chihara K. Body composition and vertebral fracture risk in female patients treated with glucocorticoid. Osteoporos Int. 2006;17(4):627-33.

34. Hodgson SF, Watts NB, Bilezikian JP, et al. American Association of Clinical Endocrinologists medical guidelines for clinical practice for the prevention and treatment of postmenopausal osteoporosis: 2001 edition, with selected updates for 2003. Endocr Pract 2003; 9(6):544-64.

35. Miller PD, Zapalowski C, Kulak CA. Bone densitometry: the best way to detect osteoporosis and to monitor therapy. J Clin Endocrinol Metab. 1999; 84(6):1867-71.

36. Cummings SR, Bates D, Black DM. Clinical use of bone densitometry: scientific review. JAMA. 2002;288(15):1889-97. 
37. Sadat-Ali M, Alelq AH, Alshafei BA, Al-Turki HA, Abujubara MA. Osteoporosis prophylaxis in patients receiving chronic glucocorticoid therapy. Ann Saudi Med. 2009;29(3):215-8.

38. Chitre MM, Hayes W. 3-year results of a member and physician intervention to reduce risk associated with glucocorticoidinduced osteoporosis in a health plan. J Manag Care Pharm. 2008;14(3):281-90.

39. Curtis JR, Westfall AO, Allison J, Becker A, Melton ME, Freeman A, Kiefe CI, MacArthur M, Ockershausen T, Stewart E, Weissman $\mathrm{N}$, Saag KG. Challenges in improving the quality of osteoporosis care for long-term glucocorticoid users: a prospective randomized trial. Arch Intern Med. 2007;167(6):591-6.

40. Peel NF, Moore DJ, Barrington NA, Bax DE, Eastell R. Risk of vertebral fracture and relationship to bone mineral density in steroid treated rheumatoid arthritis. Ann Rheum Dis. 1995;54(10):801-6

41. Sinigaglia L, Nervetti A, Mela Q, Bianchi G, Del Puente A, Di Munno O, Frediani B, Cantatore F, Pellerito R, Bartolone S, La Montagna G, Adami S. A multicenter cross sectional study on bone mineral density in rheumatoid arthritis. Italian Study Group on Bone Mass in Rheumatoid Arthritis. J Rheumatol. 2000;27(11):2582-9.

42. Johnell O, Kanis JA, Oden A, Johansson H, De Laet C, Delmas P, Eisman JA, Fujiwara S, Kroger H, Mellstrom D, Meunier PJ, Melton LJ III, O'Neill T, Pols H, Reeve J, Silman A, Tenenhouse A. Predictive value of BMD for hip and other fractures. J Bone Miner Res. 2005; 20(7):1185-94

43. De Laet C, Kanis JA, Odén A, Johanson H, Johnell O, Delmas P, Eisman JA, Kroger H, Fujiwara S, Garnero P, McCloskey EV, Mellstrom D, Melton LJ 3rd, Meunier PJ, Pols HA, Reeve J, Silman A, Tenenhouse A. Body mass index as a predictor of fracture risk: a meta-analysis. Osteoporos Int. 2005;16(11):1330-8. Epub 2005 Jun 1.

44. American College of Rheumatology Ad Hoc Committee on Glucocorticoid-induced Osteoporosis. Recommendations for the prevention and treatment of glucocorticoid-induced osteoporosis: 2001 update. Arthritis Rheum. 2001;44(7):1496-503

45. Bischoff-Ferrari HA, Willett WC, Wong JB, et al. Fracture prevention with vitamin $\mathrm{D}$ supplementation: a meta-analysis of randomized controlled trials. JAMA. 2005; 293(18):2257-64.

46. Cranney A, Horsley T, O'Donnell S, Weiler H, Puil L, Ooi D, Atkinson S, Ward L, Moher D, Hanley D, Fang M, Yazdi F, Garritty C, Sampson M, Barrowman N, Tsertsvadze A, Mamaladze $\mathrm{V}$. Effectiveness and safety of vitamin $\mathrm{D}$ in relation to bone health. Evid Rep Technol Assess (Full Rep). 2007;(158):1-235.

47. Chien KY, Chen TT, Hsu J, Pan RN, Li JH, Kuo CH, Hsu MC. Sub-maximal exercise altered the prednisolone absorption pattern. J Pharm Pharm Sci. 2010;13(1):58-66.

48. Sambrook PN. How to prevent steroid induced osteoporosis. Ann Rheum Dis. 2005;64(2):176-8.

49. World health organization collaborating centre for metabolic bone diseases, university of Shefield UK. FRAX. HWO fracture risk assessment tool. 2010 [actualizado 30 junio 2010; citado octubre 2010]. Disponible en http://www.sheffield.ac.uk/FRAX/

50. International osteroporosis foundation: FRAX WHO fracture risk assesment tool. Information and resources. [ actualizado 2009; citado octubre 2010]. Disponible en http://www.iofbonehealth. org/health-professionals/FRAX.html

51. Buckley KM, Hillner BE. A cost effectiveness analysis of calcium and vitamin D supplementation, etidronate, and alendronate in the prevention of vertebral fractures in women treated with glucocorticoids. J Rheumatol. 2003;30(1):132-8

52. Rosner AJ, Grima DT, Torrance GW, Bradley C, Adachi JD, Sebaldt RJ, Willison DJ. Cost effectiveness of multi-therapy treatment strategies in the prevention of vertebral fractures in postmenopausal women with osteoporosis. Pharmacoeconomics. 1998;14(5):559-73.

53. Lems WF, Jacobs JW, Bijlsma JW, Van Veen GJ, Houben HH, Haanen $\mathrm{CM}$, et al. Is addition of sodium fluoride to cyclical etidronate beneficial in the treatment of corticosteroid induced osteoporosis?. Ann Rheum Dis. 1997;56(6):357-63.

54. Lems WF, Jacobs WG, Bijlsma JW, Croone A, Haanen HC, Houben HH, Gerrits MI, van Rijn HJ. Effect of sodium fluoride on the prevention of corticosteroid-induced osteoporosis. Osteoporos Int. 1997;7(6):575-82.

55. Hall GM, Daniels M, Doyle DV, Spector TD. Effect of hormone replacement therapy on bone mass in rheumatoid arthritis patients treated with and without steroids. Arthritis Rheum. 1994;37(10):1499-505.

56. MacDonald AG, Murphy EA, Capell HA, Bankowska UZ, Ralston SH. Effects of hormone replacement therapy in rheumatoid arthritis: a double blind placebo-controlled study. Ann Rheum Dis. 1994;53(1):54-7.

57. Cranney A, Welch V, Adachi JD, Homik J, Shea B, SuarezAlmazor ME, et al. Calcitonin for preventing and treating corticosteroid-induced osteoporosis (Review), The Cochrane Collaboration. The Cochrane Library 2005;I:1-31.

58. Montemurro L, Schiraldi G, Fraioli P, Tosi G, Riboldi A, Rizzato G. Prevention of corticosteroid-induced osteoporosis with salmon calcitonin in sarcoid patients. Calcif Tissue Int. 1991;49(2):71-6

59. Homik J, Suarez-Almazor ME, Shea B, Cranney A, Wells G, Tugwell P. Calcio y vitamina D para la osteoporosis inducida por corticosteroides (Revisión Cochrane traducida). En: La Biblioteca Cochrane Plus, 2005

60. Cohen S, Levy RM, Keller M, Boling E, Emkey RD, Greenwald $\mathrm{M}$, et al. Risedronate therapy prevents corticosteroid-induced bone loss. A 12-month, multicenter, randomized, double-blind, placebo-controlled, parallel-group study. Arthritis Rheum. 1999;42(11):2309-18.

61. Reid DM, Hughes RA, Laan RF, Sacco-Gibson NA, Wenderoth DH, Adami S, Eusebio RA, Devogelaer JP. Efficacy and safety of daily risedronate in the treatment of corticosteroid-induced osteoporosis in men and women: a randomized trial. European Corticosteroid-Induced Osteoporosis Treatment Study. J Bone Miner Res. 2000;15(6):1006-13.

62. Kikuchi Y, et al. Effect of risedronate on high-dose corticosteroidinduced bone loss in patients with glomerular disease. Nephrol Dial Transplant. 2007;22(6): 1593-1600.

63. De Nijs RN, et al. Alendronate or Alfacalcidol in GlucocorticoidInduced Osteoporosis. N Engl J Med. 2006;355(7):675-84

64. Adachi JD, et al. Two-year effects of alendronate on bone mineral density and vertebral fracture in patients receiving glucocorticoids: a randomized, double-blind, placebo-controlled extension trial. Arthritis Rheum. 2001;44(1):202-11.

65. Sato S, Ohosone Y, Suwa A, Yasuoka H, Nojima T, Fujii T, et al. Effect of Intermittent Cyclical Etidronate Therapy on Corticosteroid Induced Osteoporosis in Japanese Patients with Connective Tissue Disease: 3Year Follow up. J Rheumatol. 2003;30(12):2673-9.

66. Adachi JD, Bensen WG, Brown J, Hanley D, Hodsman A, Josse R, Kendler DL, Lentle B, Olszynski W, Ste-Marie LG, Tenenhouse A, Chines AA. Intermittent etidronate therapy to prevent corticosteroid-induced osteoporosis. N Engl J Med. 1997;337(6):382-7.

67. Boutsen Y, Jamart J, Esselinckx W, Devogelaer JP. Primary prevention of glucocorticoid-induced osteoporosis with intravenous pamidronate and calcium: a prospective controlled 
1-year study comparing a single infusion, an infusion given once every 3 months, and calcium alone. JBone Miner Res. 2001;16(1):104-12.

68. Ringe JD, Dorst A, Faber H, Ibach K, Preuss J. Three-monthly ibandronate bolus injection offers favourable tolerability and sustained efficacy advantage over two years in established corticosteroid-induced osteoporosis. Rheumatology. 2003;42(6):743-9.

69. Saag KG, Shane E, Boonen S, Marín F, Donley DW, Kathleen A, et al. Teriparatide or Alendronate in Glucocorticoid-Induced Osteoporosis. N Engl J Med. 2007;357(20):2028-39.
70. Lane NE, Sanchez S, Genant HK, Jenkins DK, Arnaud CD. Short-term increases in bone turnover markers predict parathyroid hormone-induced spinal bone mineral density gains in postmenopausal women with glucocorticoid-induced osteoporosis. Osteoporos Int. 2000;11(5):434-42.

71. Reid DM, Devogelaer JP, Saag K, et al.: Zoledronic acid and risedronate in the prevention and treatment of glucocorticoidinduced osteoporosis (HORIZON): a multicentre, doubleblind, double-dummy, randomized controlled trial. Lancet 2009,373:1253-1263. 